\title{
A glucose monitoring system for on line estimation in man of blood glucose concentration using a miniaturized glucose sensor implanted in the subcutaneous tissue and a wearable control unit
}

\author{
V.Poitout ${ }^{1}$, D. Moatti-Sirat ${ }^{1}$, G. Reach ${ }^{1}$, Y.Zhang ${ }^{2}$, G.S. Wilson ${ }^{2}$, F. Lemonnier ${ }^{3}$, J.C. Klein ${ }^{3}$ \\ ${ }^{1}$ INSERM U341, Service de Diabétologie, Hôtel-Dieu, Paris, France \\ ${ }^{2}$ Department of Chemistry, University of Kansas, Lawrence, Kansas, USA \\ ${ }^{3}$ Centre de Morphologie Mathématique, Ecole des Mines de Paris, Fontainebleau, France
}

Summary. We have developed a miniaturized glucose sensor which has been shown previously to function adequately when implanted in the subcutaneous tissue of rats and dogs. Following a glucose load, the sensor output increases, making it possible to calculate a sensitivity coefficient to glucose in vivo, and an extrapolated background current in the absence of glucose. These parameters are used for estimating at any time the apparent subcutaneous glucose concentration from the current. In the previous studies, this calibration was performed a posteriori, on the basis of the retrospective analysis of the changes in blood glucose and in the current generated by the sensor. However, for clinical application of the system, an on line estimation of glucose concentration would be necessary. Thus, this study was undertaken in order to assess the possibility of calibrating the sensor in real time, using a novel calibration procedure and a monitoring unit which was specifically designed for this purpose. This electronic device is able to measure, to filter and to store the current. During an oral glucose challenge, when a stable current is reached, it is possible to feed the unit with two different values of blood glucose and their corresponding times. The unit calculates the in vivo parameters, transforms every single value of current into an estimation of the glucose con- centration, and then displays this estimation. In this study, 11 sensors were investigated of which two did not respond to glucose. In the other nine trials, the volunteers were asked to record every $30 \mathrm{~s}$ what appeared on the display during the secondary decrease in blood glucose. The results were analysed by comparing these readings (approximately $220 \mathrm{mea}$ surements per trial) to the changes in plasma glucose, measured every $15 \mathrm{~min}$. The Error Grid Analysis indicated that $84.1 \pm 3.6 \%$ of the measurements were in zone $\mathrm{A}$ (accurate) and $15 \pm 3.6 \%$ were in zone B (acceptable). Considering each individual trial, the differences between the displayed value and the concomitant plasma glucose concentration ranged between -1.7 and $0.69 \mathrm{mmol} / \mathrm{l}$. These excellent results were due to the absence of any significant lag between the changes in plasma glucose concentration and the changes in the result on the display. We conclude that this glucose monitoring system, based on subcutaneous sensing of glucose, is able to provide a direct on line estimation of blood glucose concentration.

Key words: Diabetes mellitus, artificial pancreas, glucose sensor, subcutaneous tissue.
The purpose of a glucose sensor is to provide a continuous estimation of glucose concentration. Potential use of glucose sensors includes continuous glucose monitoring, detection of hypoglycaemic episodes, and development of an artificial pancreas [1]. In recent years, most of the studies have focused on the development of an amperometric, enzymatic subcutaneous glucose sensor [2-12]. The principle of glucose measurement is the oxidation of glucose by glucose oxidase layered on the surface of the electrode, the generation of hydrogen peroxide and the detection of the resulting current. Indeed, the glucose concentration in the subcutanous tissue was shown to be essentially identical to plasma glucose concentration under stationary conditions [9]. Furthermore, during changes in blood glucose level observed during a glucose load, the lag between the current generated by the sensor implanted in the subcutaneous tissue and the blood glucose was shown to be usually shorter than $15 \mathrm{~min}$, when investigated in rats [9], dogs [13,14] and humans [15].

Our laboratories have developed a miniaturized glucose sensor (outer diameter, $0.45 \mathrm{~mm}$ ) which can be readily implanted in the subcutaneous tissue and replaced every 3 or 4 days [16]. This sensor has been extensively evaluated in rats and dogs, and has been shown to work for up to 10 days in rats $[9,17-19]$. The current generated by the sensor was transformed into an estimation of the concentration of glucose by using in vivo parameters, namely the sensitivity coefficient (SC, expressed in $\mathrm{nA} \cdot \mathrm{mmol}^{-1} \cdot \mathrm{l}^{-1}$ ) and 
the extrapolated background current in the absence of glucose (Io, expressed in $\mathrm{nA}$ ). It must be stressed that this method of calibration is only based on in vivo measurements [20].

In all these studies, the current was recorded during the experiment, the blood glucose was measured, and the calibration of the sensor was made a posteriori., i. e. the estimation of glucose concentration by the system was carried out by considering the results of a completed trial. In this paper, we present a method for on line calibration of the system, using a specially designed portable, battery driven, monitoring unit, and software which immediately transforms the current measured into an estimation of the glucose concentration.

\section{Subjects, materials and methods}

\section{The glucose monitoring system}

The preparation of the miniaturized glucose sensor and its in vitro characterization have been described elsewhere [16]. Briefly, the sensor consists of a platinum anode covered with teflon, except for a $1.5 \mathrm{~mm}$ cavity near its extremity, where glucose oxidase is layered. The glucose oxidase is then coated with polyurethane. A silver/silver chloride cathode is wrapped around the Teflon coating. In vitro sensitivity to glucose of sensors used in this study, determined in phosphate buffer, was $3.59 \pm 0.51 \mathrm{nA} \cdot \mathrm{mmol}^{-1} \cdot 1^{-1}$. After this determination, each sensor was sterilized with ethylene oxide and sent from the University of Kansas to Paris in a sterile package under dry conditions. Sensor implantation for in vivo testing in man was performed several weeks after sterilization.

The monitoring unit is a $6 \times 12 \times 18 \mathrm{~cm}$ battery-driven electronic device which is able to apply a $590 \mathrm{mV}$ potential between the electrodes, this potential being controlled by a digital servomechanism, and to measure, filter, and display the current. Filtering of the signal is achieved by using the algorithmic procedures of mathematical morphology [21-23]. The monitoring unit has a memory which allows the storage of up to 8,000 current values. The unit can be connected to a portable PC(Toshiba T1200XE; Toshiba Corp., Tokyo, Japan) to upload the stored values for data post-processing. The potential, the time between two measurements and the filtering characteristics can be chosen by the operator, using specially designed software. Moreover, the unit is able to display the estimation of the glucose concentration calculated from the current in real time (seebelow).

\section{Subjects}

Eleven non-diabetic human volunteers (five women and six men) participated in this study. All subjects gave their informed consent, and the study was approved by the ethical committees of INSERM and of Hotel-Dieu Hospital. The age of the subjects was $30.7 \pm 2.5$ years (mean $\pm S D$, range $20-45$ years), the $B M I$ was $22.0 \pm 1.1 \mathrm{~kg} / \mathrm{m}^{2}$ (mean $\pm \mathrm{SD}$, range 18.6-31.2).

\section{Sensor implantation}

At 18.00 hours, a 21-gauge needle was implanted $1 \mathrm{~cm}$ into the subcutaneous tissue of the forearm. The indwelling sensor was implanted through the needle, after which the needle was pulled out. No local anaesthesia was used. The sensor was then connected to a small battery applying a $590 \mathrm{mV}$ potential.

After an overnight fast, the sensor was disconnected at 08.00 hours from the battery, and connected within a few seconds to the monitoring unit. The current was measured and recorded every $30 \mathrm{~s}$.
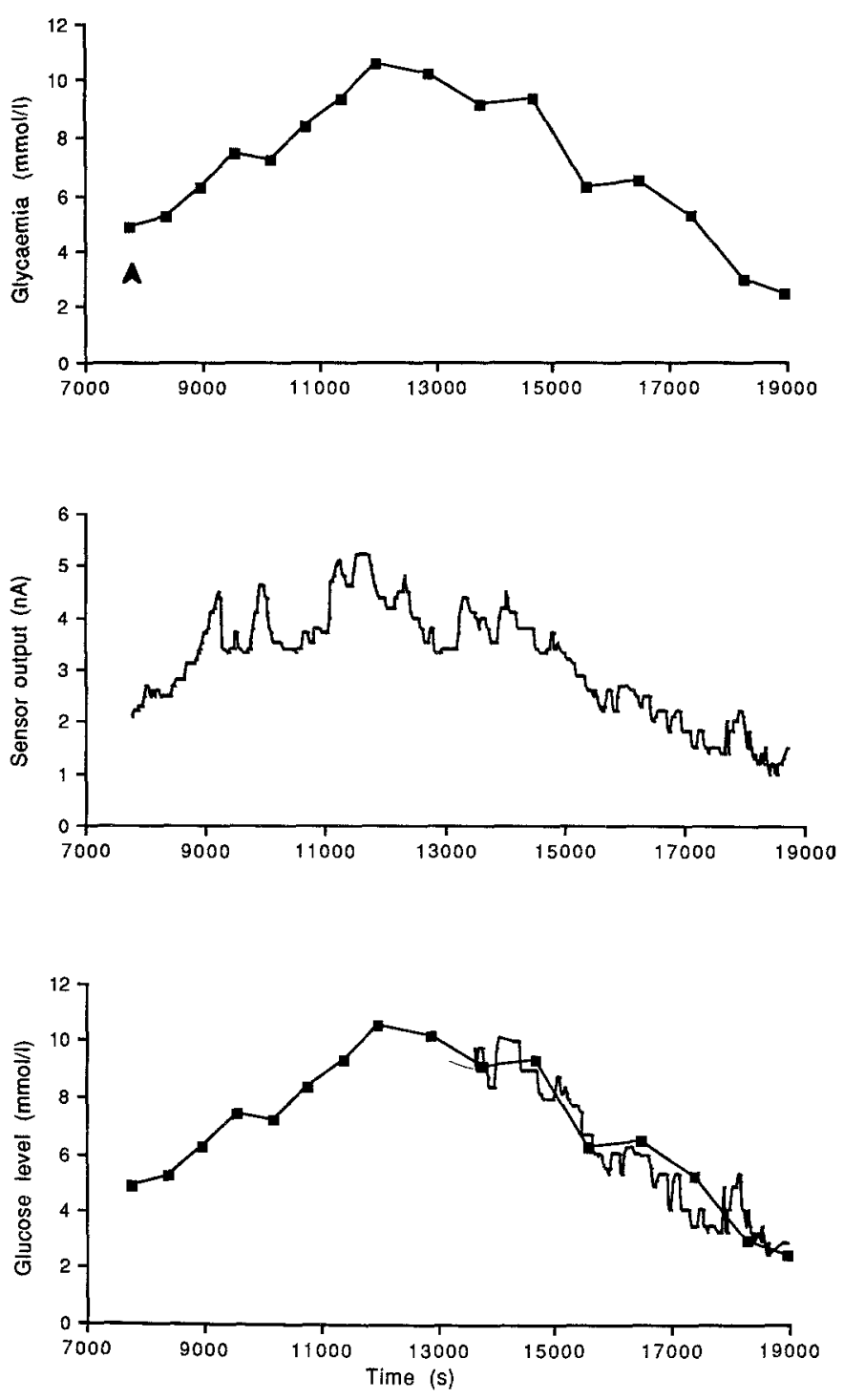

Fig.1. Results of one experiment showing the plasma glucose level following the 75-g oral glucose load (indicated by the arrow, upper curve), the current delivered by the sensor and stored every $30 \mathrm{~s}$ (middle curve), and the estimations of the glucose concentration displayed by the monitoring unit (-, lower curve) compared to the plasma glucose ( $\boldsymbol{\square}$, lower curve)

The unit was programmed to display the median of the last five values of current every $30 \mathrm{~s}$. Usually, a 2 -h run-in period was necessary to obtain a stable signal.

\section{Oral glucose tolerance test and calibration}

A 20-gauge catheter was indwelled in a forearm vein for blood sampling. At time 0 , a solution of $75 \mathrm{~g}$ of glucose dissolved in $135 \mathrm{ml}$ of water was ingested by the subjects. Plasma glucose was measured at $-10,0,10,20,30,40,50,60,75,90,105,120,135,150$ and 180 min with a Beckman Analyzer (Beckman, Fullerton, Calif., USA). The basal value of blood glucose $\left(\mathrm{G}_{1}\right)$ and its time of measurement (time $\left.{ }_{1}\right)$ were recorded. About 60 min (time $t_{2}$ ) after the ingestion of the glucose, it was observed that the current reached a stable plateau corresponding to the hyperglycaemic blood glucose value, $\mathrm{G}_{2}$. The current was considered as stable when over $5 \mathrm{~min}$ no further increase was observed. The values of $G_{1}, G_{2}, t_{1}$ and $t_{2}$ were loaded into the monitoring unit. The software then searched for the currents corresponding to $t_{1}$ and $t_{2}$ $\left(\mathrm{I}_{1}, \mathrm{I}_{2}\right)$ in the memory of the unit. By using a two-point in vivo calibra- 

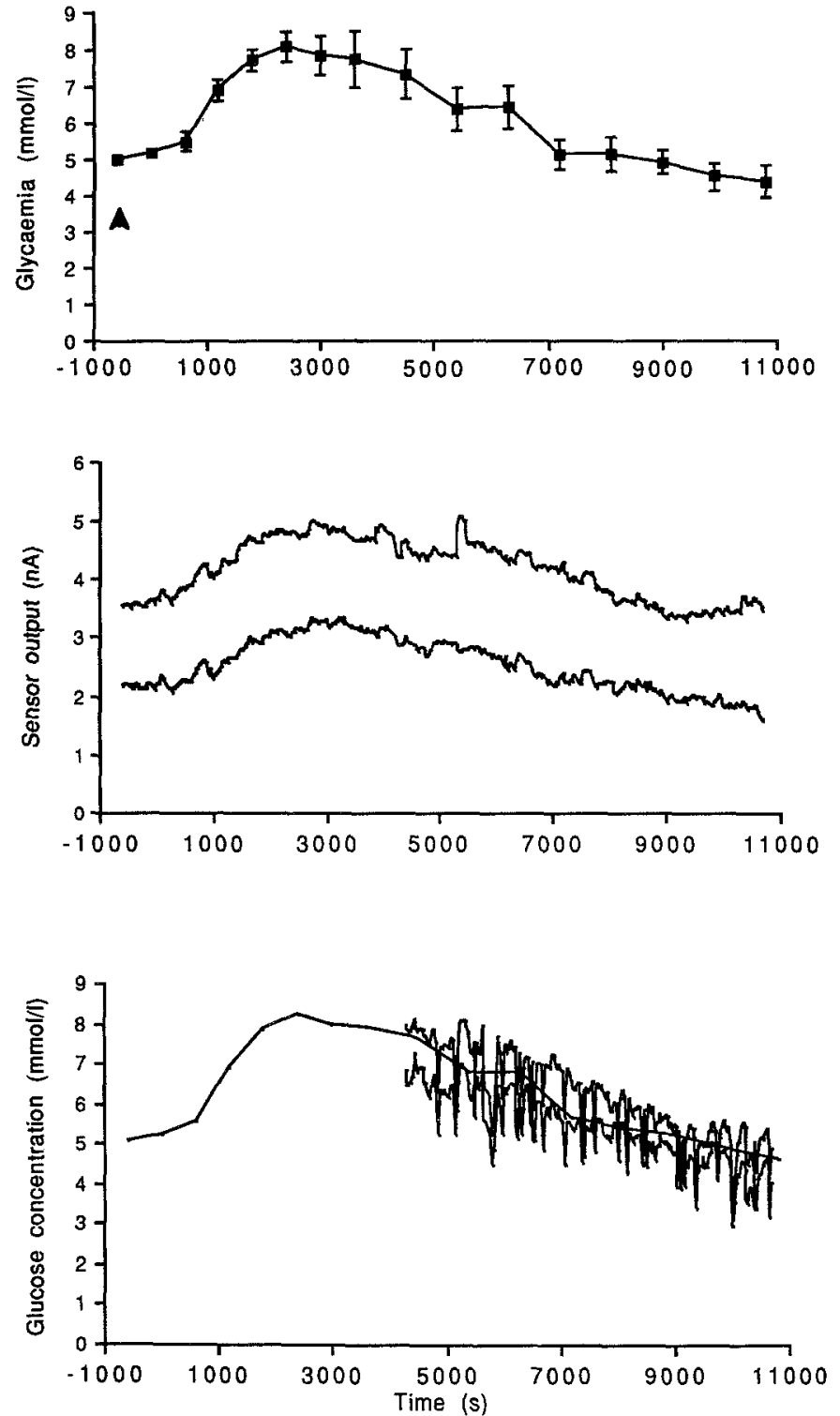

Fig. 2. Results of the nine experiments showing the mean and SEM of the plasma glucose following the 75-g oral glucose load (indicated by the arrow, upper curve), the mean + SEM and mean - SEM curves of current (middle curve), and the mean + SEM and mean SEM curves of the estimations of the glucose concentration displayed by the unit (-, lower curves), compared with the mean plasma glucose (-, lower curve)

Table 1. Error grid analysis of the nine individual experiments

\begin{tabular}{|c|c|c|c|c|c|c|}
\hline Exp. ${ }^{\circ}$ & $n$ & Zone A & Zone B & Zone C & Zone D & Zone $\mathrm{E}$ \\
\hline$\overline{1}$ & 223 & 91 & 9 & 0 & 0 & 0 \\
\hline 2 & 225 & 78.6 & 18.7 & 0 & 2.7 & 0 \\
\hline 3 & 217 & 90.8 & 7.4 & 0 & 1.8 & 0 \\
\hline 4 & 173 & 82.1 & 14.5 & 0 & 3.4 & 0 \\
\hline 5 & 214 & 86.9 & 13.1 & 0 & 0 & 0 \\
\hline 6 & 243 & 90.1 & 9.9 & 0 & 0 & 0 \\
\hline 7 & 220 & 58.2 & 41.8 & 0 & 0 & 0 \\
\hline 8 & 224 & 86.6 & 13.4 & 0 & 0 & 0 \\
\hline 9 & 262 & 92.4 & 7.6 & 0 & 0 & 0 \\
\hline Mean \pm & $4222 \pm$ & $84.1 \pm 3.6$ & $15 \pm 3.6$ & 0 & $0.9 \pm 0.4$ & 0 \\
\hline
\end{tabular}

For each experiment, the percentage of points lying in zones $A$ to $E$ is indicated tion [20], the unit then transformed every subsequent measurement of the current into an estimation of the subcutaneous glucose concentration $(\mathrm{SGC}(\mathrm{t})=(\mathrm{I}(\mathrm{t})-\mathrm{Io}) / \mathrm{SC})$. A switch on the front of the unit made it possible to display this estimation instead of the current every $30 \mathrm{~s}$. These estimations were noted by the subject over the next $2 \mathrm{~h}$. The subjects rested between the connection of the sensor to the wearable amperometric unit to the end of the oral glucose tolerance test.

\section{Statistical analysis}

For a single experiment, the estimations of subcutaneous glucose level were compared to the blood glucose from $t=60 \mathrm{~min}$ (after the calibration) to the end of the test $(t=180 \mathrm{~min})$. In order to compare the estimation of the glucose concentration by the system to the corresponding value of plasma glucose concentration every $30 \mathrm{~s}$, the latter was calculated by linear extrapolation between two measurements of actual plasma glucose, performed every $15 \mathrm{~min}$.

The two methods of glucose measurement were compared by the method of residuals [24]. As statistical analysis is insufficient for evaluating the accuracy of the estimation of glucose concentration, the clinical significance was also examined by using the "Error Grid Analysis" proposed by Clarke et al. [25]. This grid is divided in five zones, corresponding on a clinical basis to different degrees of accuracy of glucose estimations. Briefly, values of glucose concentrations in zone A are accurate, in zone B, acceptable, and in zone C, D or E unacceptable because the results would lead to an inaccurate and dangerous treatment decision.

Student's paired $t$-test was used for assessing the significance of the data.

\section{Results}

Of 11 experiments, two sensors did not respond to the increase of glucose level and therefore nine calibrations could be performed. Figure 1 presents the results of a typical experiment, and Figure 2 presents the mean and SEM of nine similar experiments. The upper curve represents the plasma glucose level. During the oral glucose tolerance test, the sensor output increased from $2.85 \pm 0.74$ to $4.19 \pm 0.83 \mathrm{nA}$ (paired $t$-test, $p=0.0002$ ) and then decreased to $2.54 \pm 0.93 \mathrm{nA}$ (middle curve of Fig. 2). The lower curve represents the mean curve of blood glucose together with the mean curve of estimated subcutaneous glucose level as displayed by the unit.

The in vivo sensitivity coefficient and background current, determined from the basal and peak value and used for the calibration procedure, were $0.45 \pm 0.09 \mathrm{nA}$. $\mathrm{mmol}^{1} \cdot \mathrm{l}^{-1}$ and $0.38 \pm 0.34 \mathrm{nA}$, respectively $(n=9)$. The in vivo sensitivity was significantly lower than that observed in vitro before the implantation $\left(3.28 \pm 0.31 \mathrm{nA} \cdot \mathrm{mmol}^{-1}\right.$. $\left.\mathrm{l}^{-1}, p=0.01, n=9\right)$ and than that determined in vitro immediately after explantation $\left(1.17 \pm 0.31 \mathrm{nA} \cdot \mathrm{mmol}^{-1} \cdot \mathrm{l}^{-1}\right.$, $p=0.01, n=9$ ).

Analysis of the results of the experiment shown in Figure 1 through the Error Grid Analysis is shown in Figure 3, and Table 1 represents the results of the nine experiments. $99.1 \pm 3.6 \%$ of the estimations were in zone A or B (considered as accurate (A) or acceptable (B). Figure 4 represents the results of the same experiment through the method of residuals. The differences between the displayed value and the concomitant plasma glucose concentration were plotted against the mean of the two values. The three horizontal lines represent, respectively, the 
mean, the mean +2 SD and the mean -2 SD of these differences. In this trial, they were found to be, respectively, $0.18,1.72$ and $-1.36 \mathrm{mmol} / \mathrm{l}$. This indicates that in this trial, $95 \%$ of the 173 values differed from the actual plasma glucose concentration by less than $1.72 \mathrm{mmol} / \mathrm{l}$.

Table 2 gives the data for the nine trials. The mean of the differences ranged from -1.7 to $0.69 \mathrm{mmol} / \mathrm{l}$ (mean \pm SEM of the nine trials: $-0.31 \pm 0.28$ ), and the values for $2 \mathrm{SD}$ ranged from 0.94 to $2.62 \mathrm{mmol} / \mathrm{l}$ (mean $\pm \mathrm{SEM}$ of the nine trials: $1.67 \pm 0.16$ ).

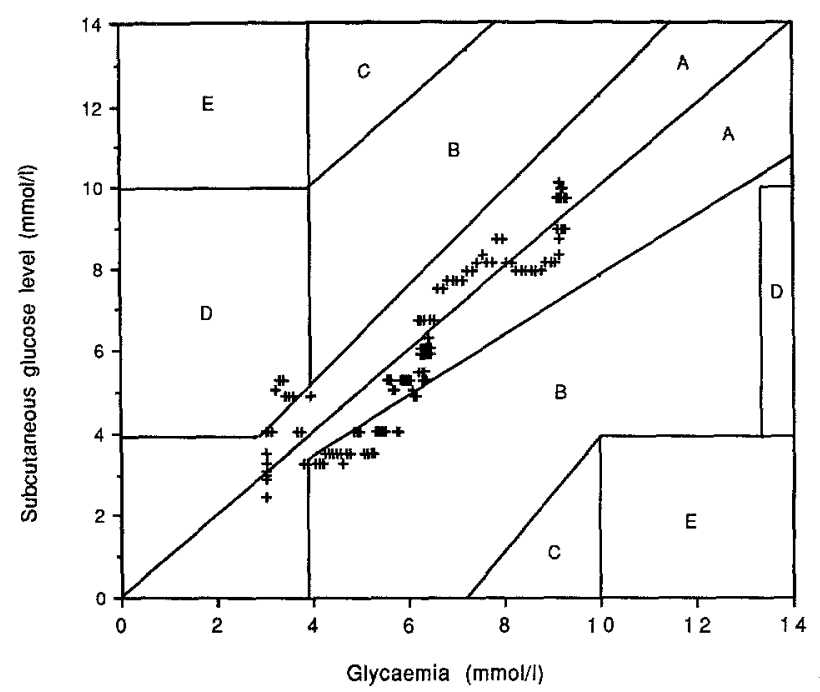

Fig. 3. Error Grid Analysis of the experiment shown in Figure 1. The estimations of the glucose concentration by the glucose monitoring system are plotted against the actual plasma glucose, as determined between two measurements by linear extrapolation

\section{Discussion}

Although the development of self monitoring of blood glucose (SMBG) can be considered as a milestone in the history of diabetes therapy [26], it has two major drawbacks related to its discontinuous nature as Type 1 (insulin-dependent) diabetic patients are usually not willing to perform more than 3 to 4 daily measurements: First, it cannot be used as a warning system of severe hypoglycaemic episodes, which can occur between two measurements. This is a subject of concern, especially in view of the recently recognized syndrome of hypoglycaemia unawareness [27]; secondly, the problem of nocturnal hypoglycaemia, the frequency of which was recently pointed out [28], cannot be resolved by conventional SMBG. This demonstrates the interest in developing a continuous glucose monitoring system.

A glucose monitoring system must consist of two parts: a glucose sensor and an electronic device. The glucose sensor must be either totally implantable, or small enough to be readily replaced by the patient himself. Similarly, the electronic control unit must be miniaturized in order to be wearable in normal daily life. It must be pointed out that the functions of such a unit are multiple: controlling the potential of the working electrode, sampling, filtering, storing and processing the current, and transforming the current generated by the sensor into an estimation of a glucose concentration. In addition, it is essential that the result is given in real time, i. e. the result is still relevant when it becomes available. This implies that the glucose sensor has a short response time, but also that the changes in blood glucose concentration are rapidly transmitted to the subcutaneous monitoring site. Indeed, the aim of the

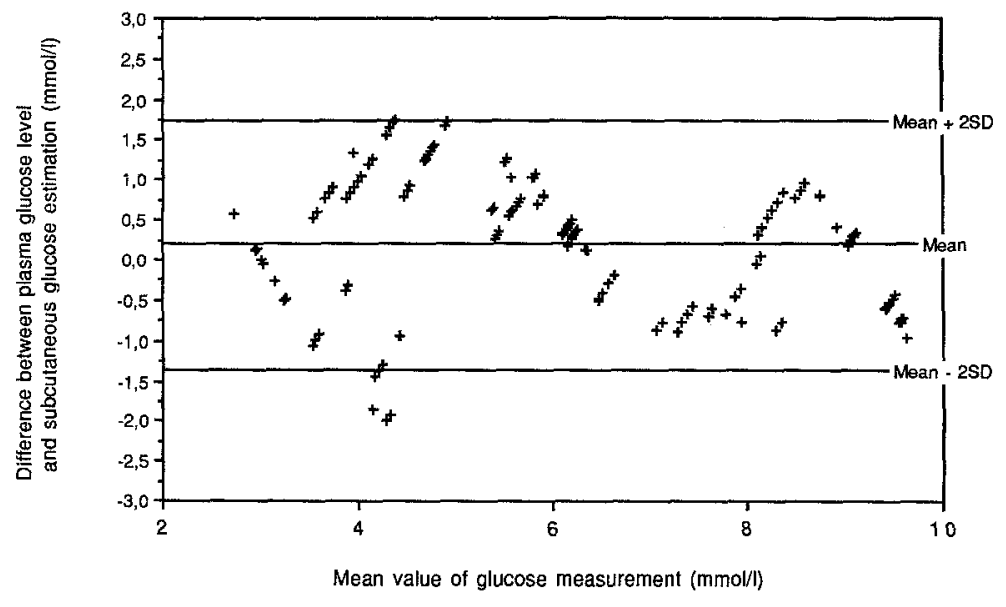

Fig. 4. Analysis of the data presented in Figure 2 through the method of residuals. Each difference between the plasma glucose level and the estimation of the glucose concentration by the unit is plotted against the mean individual value of glucose level

Table 2. Analysis of the data through the method of residuals

\begin{tabular}{llllllllll}
\hline Exp. $\mathrm{n}^{\circ}$ & 1 & 2 & 3 & 4 & 5 & 6 & 7 & 8 \\
\hline$n$ & 223 & 225 & 217 & 173 & 214 & 243 & 220 & 224 & 262 \\
Mean difference & 0.57 & -1.3 & -0.76 & 0.18 & -0.36 & -0.54 & -1.7 & 0.69 & 0.46 \\
2SD & 2.62 & 1.7 & 1.2 & 1.54 & 1.88 & 1.6 & 2.02 & 1.5 & 0.94 \\
SEM & 0.09 & 0.06 & 0.04 & 0.06 & 0.06 & 0.05 & 0.07 & 0.05 & 0.03 \\
$t$ & 6.5 & -22.9 & -18.7 & 3.1 & -5.6 & -10.5 & -25 & 13.8 & 15.8 \\
\hline
\end{tabular}

For every experiment are indicated the number of comparisons, the mean difference between plasma glucose and subcutaneous glucose concentration, the SD and the SEM of the difference, and the paired $t$ value of the comparisons. All $p$ values are $<0.01$ 
system is to provide the patient with continuous access to his/her blood glucose concentration, which is the needed relevant information.

This paper provides the evidence that our glucose monitoring system meets these requirements. First, the glucose sensor, the function of which was extensively investigated both in vitro and in vivo $[9,16-20]$, can be readily implanted in the subcutaneous tissue without anaesthetic. Secondly, a wearable electronic unit was designed to fulfill all the requirements outlined above. More specifically, the system contains built-in software for transforming the current into an estimation of glucose concentration. Signal filtering is achieved by a mathematical morphology method [21-23]. In this study, a rather crude processing of the signal (median of the five last values) was used, which proved to be sufficient in these preliminary trials, although noise was far from being completely eliminated. However, the novel use of signal processing through mathematical morphology procedures should provide the basis for implementing in the future better filtering algorithms. Finally, the architecture of the control unit was designed to be compatible with its further miniaturization to the size of a wrist-watch.

The aim of this study was to develop and to validate a procedure for on line calibration of the system. For this purpose, eleven different sensors were implanted. Two of them did not generate a sufficient increase in the current during the glucose load. Investigations are in progress to understand why some sensors are "blind" to glucose. With the nine other sensors, the current increase was used to calibrate the system under a procedure based only on the changes in the current. When, during the course of a glucose load (oral glucose tolerance test in these normal subjects), the current was estimated to have reached a stable peak value, the blood glucose concentration was determined which was used for calibrating the system. The display was then switched to a "glucose mode" where the estimation of glucose concentration appeared on the liquid crystal display of the control unit during the secondary decrease of blood glucose concentration. It must be pointed out that this calibration procedure, as it is based only on the current changes, could ultimately be performed automatically by the system.

Data analysis through the method of residuals indicated that statistically the displayed value was significantly different from the concomitant plasma glucose concentration. However, this was due to the very high number of analysed measurements. By contrast, the Clarke analysis of the data, which is clinically-oriented, indicated that $99 \%$ of these data appearing on the display can be considered as accurate $(84 \%)$ or acceptable $(15 \%)$ when compared to the actual blood glucose concentration. This demonstrates that this system, using a miniaturized glucose sensor implanted in the subcutaneous tissue, gives at any time a reliable estimation of blood glucose concentration. It can be stressed that the calibration was performed by using a slight increase in plasma glucose (from $5.19 \pm 0.11$ to $8.06 \pm 0.4 \mathrm{mmol} / 1$ ), similar to those observed in a well-controlled Type 1 diabetic patient.

As a conclusion, this paper describes an original method for calibrating on line a glucose monitoring sys- tem on the basis of minor changes in blood glucose concentration, such as those which would be commonly observed in well-equilibrated Type 1 diabetic patients. Calibrating the sensor on the basis of an "in vivo" sensitivity is essential since the sensitivity in vivo is different from that determined under in vitro conditions, as shown in this study, confirming all our previous reports $[9,17-20]$ and reports by others $[13,14]$. The originality of this method is that it is based on the changes in the current, and that it should therefore provide the basis for a fully automated procedure. The software could be improved to be able to recognize a current stability, ask the patient to measure a blood glucose concentration and enter the data, and to repeat the procedure a second time to provide the second set of values necessary for achieving the two-point calibration. In this study, the calibration procedure was performed $14 \mathrm{~h}$ after the sensor implantation, the sensor being polarized in the meantime with a battery: the connection to the electronic control unit would not have been comfortable, due to its present size. This study was not aimed to describe the lifespan of the sensor, and further investigations are needed to determine its long-term stability. However, the lifespan of a similar sensor was evaluated in rats and this sensor was proved to function satisfactorily at least 10 days after implantation in the subcutaneous tissue [18]. Finally, these results indicate that the kinetics of the changes in subcutaneous glucose concentration in man are extremely rapid. This could have important implications for the choice of the subcutaneous tissue as a site for implantation of a closed-loop insulin delivery system, including a bioartificial pancreas.

Acknowledgements. This study was supported by Institut National de la Santé et de la Recherche Médicale (grant CRE 89-90-14), National Institutes of Health (USA, grant $n^{\circ} \mathrm{D}$ and $\mathrm{K} 30718$ ), Hoechst AG, Frankfurt. Aide aux Jeunes Diabétiques (grant to VP).

\section{References}

1. Reach G (1993) Continuous glucose monitoring with a subcutaneous sensor. Rationale, requirements and achievements, and prospectives. In: Alberti KGMM, Krall LP (eds) The diabetes annual, Elsevier Science Publishers BV, Amsterdam, In press

2. Shichiri M, Yamasaki Y, Kawamori R, Abe H (1982) Wearable artificial pancreas with needle type glucose sensor. Lancet II: $1129-1131$

3. Abel P, Müller A, Fischer U (1984) Experience with an implantable glucose sensor as a prerequisite of an artificial beta cell. Biochem Biomed Acta 43: 577-588

4. Claremont DJ, Sambrook IE, Penton C, Pickup JC (1986) Subcutaneous implantation of a ferrocene-mediated glucose sensor in pigs. Diabetologia 29: $817-821$

5. Pickup JC, Shaw GW, Claremont DJ (1989) In vivo molecular sensing in diabetes mellitus: an implantable glucose sensor with direct electron transfer. Diabetologia 32: $213-217$

6. Pickup JC (1985) Biosensors: a clinical perspective. Lancet Ir: 817-820

7. Matthews DR, Bown E, Beck TW et al. (1987) An amperometric needle-type glucose sensor tested in rats and man. Diabetic Med 5:248-252

8. Koudelka M, Rohner-Jeanrenaud F, Terrettaz J, BobbioniHarsch E, de Rooij NF, Jeanrenaud B (1991) In-vivo behaviour of hypodermically implanted microfabricated glucose sensors. Biosens Bioelectron 6: 31-36 
9. Velho G, Froguel Ph, Thévenot DR, Reach G (1988) In vivo calibration of a subcutaneous glucose sensor for determination of subcutaneous glucose kinetics. Diab Nutr Metab 1: 227-233

10. Ege H (1989) A needle-shaped glucose sensor using an aqueous polyurethane dispersion for membrane dormation and for immobilization of glucose oxidase. Artif Org 13: 171 (Abstract)

11. Mascini M, Moscone D, Palleschi G(1989) Needle sensor for glucose measurement. Artif Org 13: 173 (Abstract)

12. Kerner W, Brueckel J, Zier Het al. (1989) Glucose measurement in subcutaneous tissue. Artif Org 13: 173 (Abstract)

13. Fischer U, Ertle R, Abel P et al. (1987) Assessment of subcutaneous glucose concentration: validation of the wick technique as a reference for implanted electrochemical sensors in normal and diabetic dogs. Diabetologia 30: 940-945

14. Rebrin K, Fischer U, Hahn von Dorsche H, von Woetke T, Abel $P$, Brunstein E (1992) Subcutaneous glucose monitoring by means of electrochemical sensors: fiction or reality? J Biomed Eng 14: 33-40

15. Jannson PA, Fowelin J, Smith U, Lönnroth P (1988) Characterization by microdialysis of intracellular glucose level in subcutaneous tissue in humans. Am J Physiol 255: E218-E220

16. Bindra DS, Zhang Y, Wilson GS et al. (1991) Design and in vitro studies of a needle type glucose sensor for subcutaneous monitoring. Anal Chem 63: 1692-1696

17. Poitout V, Moatti $D$, Velho $G$ et al. (1991) In vitro and in vivo evaluation in dogs of a miniaturized glucose sensor. Trans Am Soc Artif Int Org 37: M298-M300

18. Moatti-Sirat D, Capron F, Poitout V et al. (1992) Towards continuous glucose monitoring: in vivo evaluation of a miniaturized glucose sensor implanted for several days in rat subcutaneous tissue. Diabetologia 35: 224-230

19. Poitout V, Moatti-Sirat D, Reach G (1992) Calibration in dogs of a subcutaneous miniaturized glucose sensor using a glucose meter for blood glucose determination. Biosens Bioelectron 7 : $587-592$

20. Velho G, Froguel Ph, Thévenot DR, Reach G (1989) Strategies for calibrating a subcutaneous glucose sensor. Biochim Biomed Acta 48: 957-964
21. Serra J (1982) Morphology for grey-tone function. In: Serra J (ed) Image analysis and mathematical morphology - Vol 1. Academic Press, London, pp 474-476

22. Serra J (1982) Dilation and filtering for numerical function. In: Serra J (ed) Image analysis and mathematical morphology - Vol 2. Academic Press, London, pp 181-202

23. Serra J (1982) Alternating sequential filters. In: Serra J (ed) Image analysis and mathematical morphology - Vol2. Academic Press, London, pp 203-216

24. Bland JM, Altman DG (1986) Statistical methods for assessing agreement between two methods of clinical measurement. Lancet I: 307-310

25. Clarke WL, Cox D, Gonder-Frederick LA, Carter W, Pohl SL, (1987) Evaluating clinical accuracy of systems for self-monitoring of blood glucose. Diabetes Care 5: 622-627

26. Tattersall RB (1985) Self-monitoring of blood glucose - 1978 1984. In: Alberti KGMM, Krall LP (eds) The diabetes annual, Elsevier Science Publishers BV, Amsterdam, pp 162-177

27. Berlin I, Grimaldi A, Landault C et al. (1988) Lack of hypoglycaemic symptoms and decreased beta-adrenergic sensitivity in insulin-dependent diabetic patients. J Clin Endoc Metab 66: 273278

28. The DCCT Research Group (1991) Epidemiology of severe hypoglycaemia in the diabetes control and complications trial. Am J Med 90: 450-459

Received: 21 December 1992

and in revised form: 15 March 1993

Dr. G. Reach

INSERM U341

Service de Diabétologie

Hôtel-Dieu 1, Place du Parvis Notre Dame

F-75004 Paris

France 